\title{
Load monitoring on Pilates training: a study protocol for a randomized clinical trial
}

\author{
Allysiê Priscilla de Souza Cavina ${ }^{1,2^{*}}$, Eduardo Pizzo Junior ${ }^{3}$, Aryane Flauzino Machado ${ }^{1}$, Taíse Mendes Biral ${ }^{1}$, \\ Carlos Marcelo Pastre ${ }^{4}$ and Franciele Marques Vanderlei ${ }^{4}$
}

\begin{abstract}
Background: Currently there are campaigns to raise the awareness of the need to practice physical exercise with several objectives, mainly as a preventive measure. The Pilates method is a form of therapeutic exercise for maintaining and improving health. However, despite being popular, there is still no scientific evidence on the standardization and progression of the method. Therefore, the purpose of this study was to develop a protocol to monitor the progression of daily Pilates loads between the basic, intermediate, and advanced levels, as well as to analyze the effects of the method on psychometric, cardiorespiratory, and autonomic measures.
\end{abstract}

Methods/design: In total, 54 healthy men underwent 36 sessions of Pilates mat work. Before each training session, cardiorespiratory measures, pain (visual analogue scale), and a psychometric questionnaire were collected. Heart rate (HR), subjective perception of effort (SPE), and RR intervals were measured during the sessions and used later in the analysis of the progression of training load by monitoring the internal training load and heart rate variability. At the end of the sessions, cardiorespiratory measures, the visual analogue scale, and the psychometric questionnaire were measured again. After 15 min of rest, the final HR measurement was made and the participants noted the effort on the SPE scale. The psychometric, cardiorespiratory, and autonomic measures were evaluated before and after each of the 36 training sessions.

Discussion: This is a parallel randomized clinical trial of standardized Pilates training, with the aim of estimating training loads and measuring the efficacy of Pilates through clinical, cardiorespiratory, and autonomic outcomes. The protocol can easily be reproduced and could be used to support professionals in prescribing the method.

Trial registration: ClinicalTrials.gov, NCT03232866. Registered on 28 July 2017.

Keywords: Exercise movement techniques, Exercise, Monitoring, Physiologic, Heart rate, Autonomic nervous system

\section{Background}

The Pilates method is an alternative therapeutic exercise for protecting against risk factors and promoting health [1]. However, only one literature review investigates the Pilates method [2]. The methodological quality is low, which demonstrates the lack of standardization of the method and the load progression as a form of physical

\footnotetext{
* Correspondence: lysi_cavina@hotmail.com

${ }^{1}$ Postgraduate Program in Physiotherapy, Universidade Estadual Paulista (FCT/UNESP), Presidente Prudente, SP, Brazil

${ }^{2}$ Faculdade de Ciências e Tecnologia - FCT/UNESP, Departamento de Fisioterapia, Rua Roberto Simonsen, 305 - Cidade Universitária, Presidente Prudente, SP 19060-900, Brazil

Full list of author information is available at the end of the article
}

training [2]. Thus, for the safe prescription of resistance exercises, such as the Pilates method, physiological variables, such as heart rate (HR) [3] and cardiac autonomic modulation $[4,5]$, and subjective variables, such as subjective perception of effort (SPE) [6], need to be monitored.

Thus, there is a need to identify simple and low-cost methods for controlling the intensity of exercises, due to the adaptations made by the psychobiological system through training, such as improvements in exercise tolerance and physical performance [7]. It would be beneficial to use SPE together with HR to quantify the training intensity of the Pilates method, as well as to use cardiac autonomic modulation to assess the oscillations

(c) The Author(s). 2019 Open Access This article is distributed under the terms of the Creative Commons Attribution 4.0 International License (http://creativecommons.org/licenses/by/4.0/), which permits unrestricted use, distribution, and 
of the cardiac autonomic nervous system during training, since heart rate variability (HRV) is considered an early and sensitive indicator of health impairment. HRV indicates the adaptability of cardiac autonomic control to a stimulus $[8,9]$.

Although the Pilates method is becoming more widespread and has shown promising clinical and functional outcomes in rehabilitation [10,11], quality clinical trials are needed to analyze the intensity of the training. The results of such trials may offer greater support for tools that can assist in the prescription and load progression of Pilates practice, while demonstrating the effects of the method on the cardiovascular system and autonomic control.

Therefore, the objective of this protocol is to monitor the daily load progression from the basic and intermediate levels to the advanced level by measuring HR, SPE, and HRV for 12 weeks, as well as to analyze the effect of the method on psychometric, cardiorespiratory, and autonomic measures. It is hypothesized that the protocol proposed by the study can be prescribed clinically, proving that SPE, HR, and HRV are useful tools for monitoring the progression of Pilates training loads. Furthermore, SPE is expected to have a good correlation with HR and clinical relevance, thus giving practitioners two simple low-cost monitoring tools. In addition, it is hypothesized that the psychometric, cardiorespiratory, and cardiac autonomic modulation measures will have improved after 12 weeks of training.

\section{Methods/design}

\section{Study design}

A parallel randomized clinical trial is being conducted at the Center for Studies and Assistance in Physiotherapy and Rehabilitation of the Universidade Estadual Paulista (FCT/ UNESP), Presidente Prudente, São Paulo, Brazil. The trial was registered at ClinicalTrials.gov (NCT03232866) and approved by the research ethics committee of FCT/UNESP (protocol 061942/2017). The study protocol follows the SPIRIT 2013 checklist (Standard Protocol Items: Recommendations for International Trials) [12] (Additional file 3) and TIDieR (Template for Intervention Description and Replication) [13], indicating that information about and the quality of the reports of the interventions are well described [14]. Prior to participation, the volunteers received oral and written instructions and signed a consent form agreeing to participate in the study. All personal data will remain confidential. Note that, although very unlikely, participants who suffer any kind of injury as a result of participation will receive a free medical evaluation and physiotherapy treatment.

Participants were divided into two groups: the Pilates group and the control group. Participants in the Pilates group underwent 12 weeks of Pilates exercises for three times a week, for a total of 36 sessions. Each session lasted for approximately $60 \mathrm{~min}$. During this training period, participants were required to pass through the three levels of the Pilates method: basic, intermediate, and advanced. The control group were asked to maintain their current level of daily activities without taking any type of exercise training. A flowchart for the study and group composition are shown in Fig. 1.

\section{Participants}

A total of 54 healthy male volunteers were recruited from the local university (UNESP) through pamphlets, online media, personal invitations, telephone calls, and SMS messages. These procedures were recommended by Treweek et al. [15] as strategies to improve participant recruitment.

The inclusion criteria were: male; 18 to 35 years of age; healthy (self-report); not having practiced Pilates prior to the study; not a smoker or an alcoholic; not presenting with a metabolic, cardiac, or endocrine disorder; with no arrhythmia; not using drugs that influence the autonomic modulation of the heart; and without physical limitations such as musculoskeletal, inflammatory, or neurological diseases that would prevent them from doing Pilates.

Individuals who suffered from a musculoskeletal injury during training, those who attended less than $85 \%$ of training sessions, those who failed to progress between the three levels of the Pilates method, those who were unable to respond adequately to subjective scales, and those with errors in the captured RR intervals were excluded from the study. Participants were instructed to maintain their daily dietary routine, to abstain from taking anti-inflammatory medications and analgesics, and not to perform any other type of exercise during the intervention period. These requests were reinforced during the training period and monitored by self-report [16].

\section{Randomization}

Prior to randomization, baseline data were collected from participants who meet the eligibility criteria and had signed the informed consent form. To maintain the blinding, randomization by group (Pilates group or control group) was performed by another researcher not involved in the recruitment using a computer-generated randomization schedule.

\section{Details of procedures Study outline}

The evaluations were carried out at FCT/UNESP, in a room with controlled temperature and humidity, at the same time of day. For the initial evaluation, after completing the personal information, the participants completed a psychometric questionnaire [17]. Once the anthropometric 


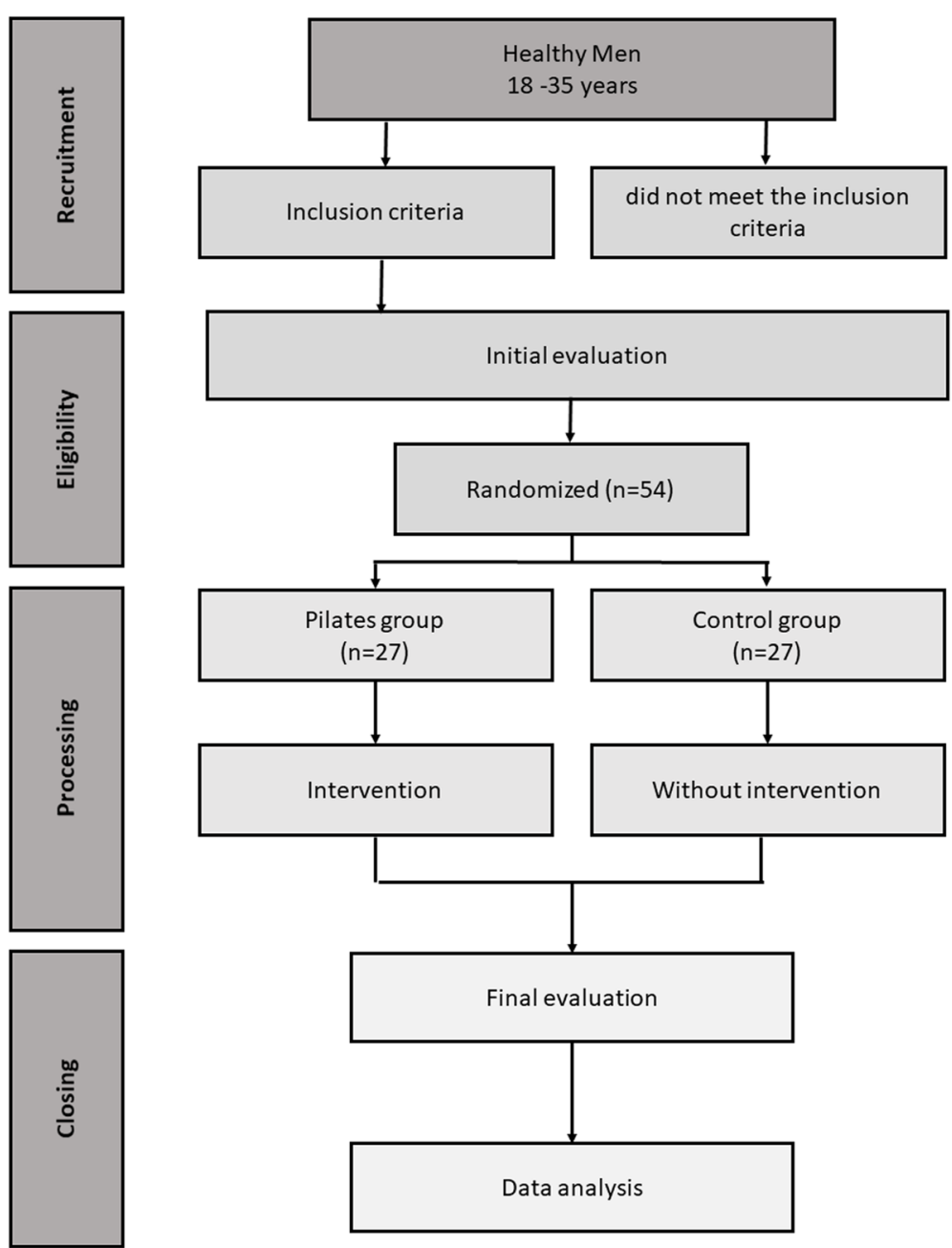

Fig. 1 Flowchart of the study

metrics had been collected for each participant, their body mass index was calculated.

In addition, systolic and diastolic blood pressure, HR, respiratory rate, and oxygen saturation were measured. Then, the participants were sent to a quiet room where a cardiofrequency meter (V800, Polar Electro Oy, Kempele, Finland) recorded their HR beat to beat for $20 \mathrm{~min}$ for later analysis of HRV.

The Pilates training began after a week with three familiarization sessions. At the start of each training session, cardiorespiratory measures (systolic blood pressure, diastolic blood pressure, respiratory rate, $\mathrm{HR}$, and oxygen saturation) and pain (visual analogue scale) were measured, and a psychometric questionnaire was completed, as the basal control. To measure the progression of load, HR, SPE, and vagal indices of HRV were used. These metrics were collected during the exercises. HR was measured and recorded on the
SPE scale every 5 minutes, and the RR intervals were recorded during the whole session. At the end of each session, the cardiorespiratory metrics and pain (visual analogue scale) were measured, and the psychometric questionnaire was completed. After $15 \mathrm{mi}-$ nutes of rest, the volunteers underwent the final HR measurements and completed the SPE scale again. The sessions are shown in Fig. 2.

At the end of the 36 Pilates training sessions, the final evaluation was carried out. This included cardiorespiratory measures, the psychometric questionnaire, and HRV. The control group underwent only the initial and final evaluations, like the Pilates group.

\section{Pilates exercises}

The exercises are shown in Additional file 1. The exercise sequences were divided in different ways to make the training more varied and to avoid dropouts 


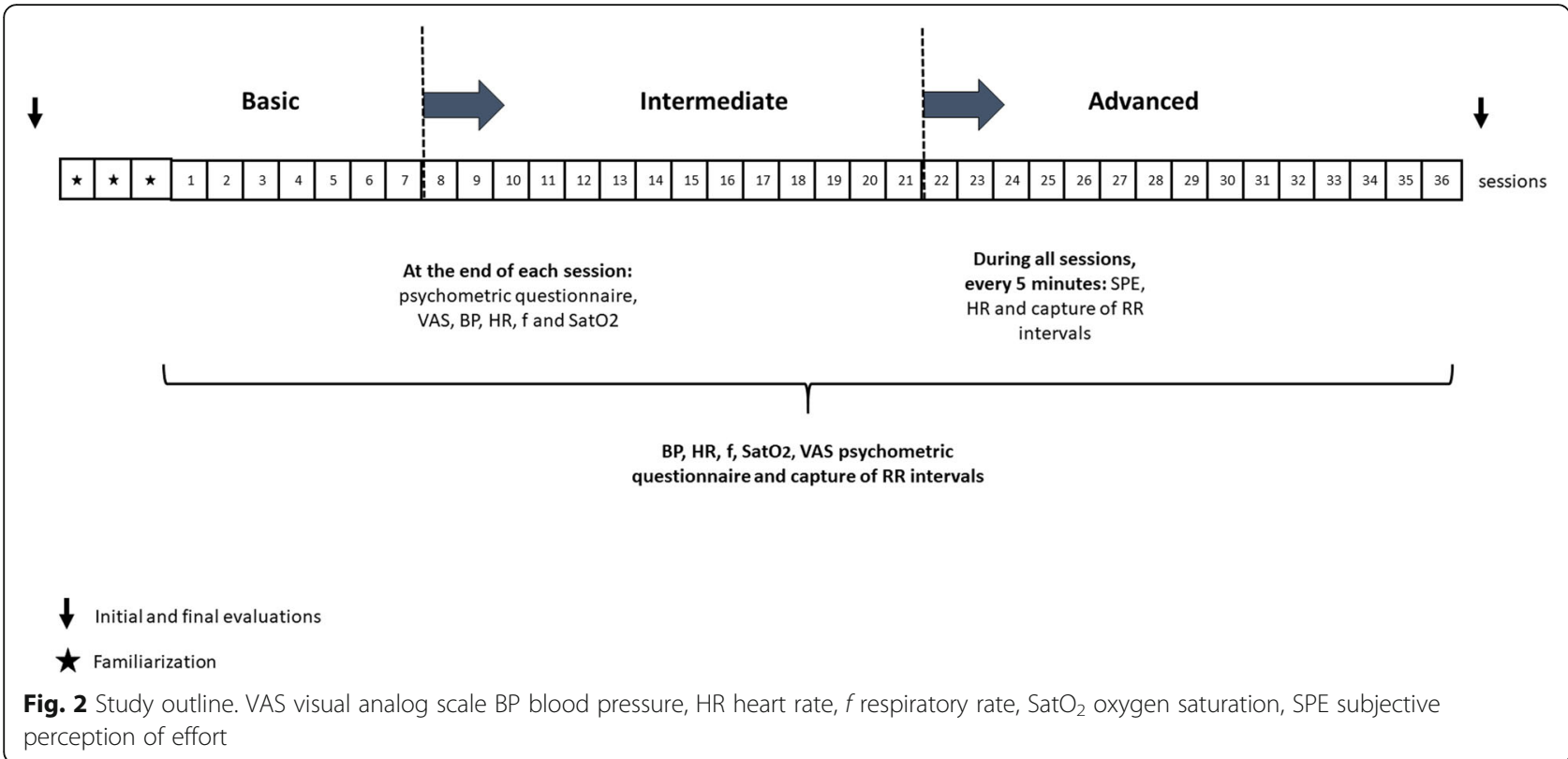

(Additional file 2). The exercise schedule was produced by us based on an extensive bibliographical survey of Pilates exercises and their progression. We considered the degree of difficulty of each exercise as well as the dependence between volume and intensity.

\section{Primary outcome}

Quantification of the internal training load was evaluated in each training session with the three monitoring tools: SPE [6], HR [18], and HRV [18]. For the monitoring to be considered successful, two of the three monitoring tools must yield significant results. In addition, the correlation between SPE and HR should be moderate to high so that they can be used in clinical practice as low cost and easily applicable tools.

The SPE consists of a scale proposed by Borg where the participants choose the descriptor and number that best represents their psychophysiological stress in the training session [6]. It is a scale from 6 to 20 points, where 6 means "no effort" and 20 means "maximum effort." To avoid biased responses, the question was standardized as: "From 6 to 20 points, how do you rate your perception of effort now?" All responses were recorded in individual records. The participants had previously been made familiar with the scale [6].

Prior to the sessions, participants were told how to estimate their SPE. It was explained to them that during the exercises it was necessary to perceive how much effort they were using so that every 5 minutes during a session they could estimate the SPE according to their individual perception for how exhausting the exercises were.
In addition, $15 \mathrm{~min}$ after the end of each session, the participants estimated the SPE again [6]. This final SPE was multiplied by the duration of the session in minutes, and the product represents the internal load in arbitrary units. The intensity of the session was classified into three zones according to the Borg scale used by Moreira et al. [19].

Quantification of the internal load training using the HR was performed using the TRIMP method. This evaluates the volume and intensity of the session through specific scores in each training zone, as proposed by Edwards [18, 20].

In addition to these methods for monitoring the training load, HRV was analyzed. The weekly mean of rMSSD (rMSSDmean) expressed in milliseconds and the weekly intra-individual coefficient of variation of rMSSD (rMSSDcv) expressed as a percentage will be used [21, 22]. These indices will be transformed to logarithms to mitigate outliers and simplify the analysis.

\section{Secondary outcomes}

The HRV will be calculated from the series of RR intervals captured by the cardiofrequency monitor using linear methods. It will be analyzed in the time and frequency domains. A Poincaré plot will be used. All HRV indices will be calculated by Kubios HRV software version 3.1.0.

The RR interval series was recorded before and after the 12 weeks of training. There were $1000 \mathrm{RR}$ intervals in each data set. In addition, daily tracking of each session will be analyzed. Stretches of 256 consecutive RR 
intervals were recorded every 5 minutes. In both analyses, the most stable stretches were used to avoid any bias in the analysis and data interpretation [5]. The time series of RR intervals will undergo moderate digital filtering using Kubios, supplemented by manual filtering to eliminate premature ectopic beats and artifacts. Only series with more than $95 \%$ of sinus beats will be included in the final analysis [23].

The time domain (rMSSD and standard deviation of all RR intervals) [24], frequency (low and high) $[23,25,26]$, and Poincaré plot (standard deviation of data from $x=y$ and standard deviation of data from the line orthogonal to $x=y$ that passes through the mean value of the data) $[23,27,28]$ HRV indices will be used for the analyses before and after the 12 weeks of training. Only the time domain indices and the Poincaré plot will be used for daily tracking.

HR [23], respiratory rate measurements [29], and oxygen saturation [30] were captured. Blood pressure [31] was measured. A subjective pain assessment was obtained through a visual analog pain scale [32, 33]. Volunteers were asked to fill out a psychometric questionnaire [17] before and after the training period, as well as before all training sessions.

All these outcomes were collected in the initial evaluation and in the final evaluation after the 12 weeks of training, in addition to before and after each Pilates session. Table 1 lists outcome collection time points.

\section{Masking and blinding}

Baseline outcomes were collected prior to randomization. The final evaluation was conducted by trained evaluators blinded to group allocation. In addition, data were collected throughout the Pilates training period by independent evaluators. The study statistician will also be blinded. In this study, it was not possible to blind either the participants or the therapist who administered the Pilates sessions.

\section{Sample size calculation}

The sample calculation was carried out based on Barbosa et al. [34] with the rMSSD index. The clinically relevant difference was $19,02 \mathrm{~ms}$ and the standard deviation $23,94 \mathrm{~ms}$. For a two-tailed hypothesis test, the significance level for the sample calculation was $5 \%$ and the power of the test was $80 \%$. The sample size was calculated to be 25 per group. To account for withdrawals, the sample size was increased by $10 \%$ to give 27 volunteers per group.

\section{Statistical analysis}

Descriptive statistics will be used, and the results presented as means, errors, standard deviations, percentages, and absolute numbers. The normality of the data will be evaluated through a Shapiro-Wilk test. In the comparison of the population profile and the cardiorespiratory, autonomic, and psychometric measures in the initial and final evaluations, the paired $t$ test will be used for normal data or the Wilcoxon test for non-normal data. In the comparison between the groups, the unpaired $t$ test or Mann-Whitney test will be used depending on the normality of the data, with the significance level set at $p<0.05$. Differences based on magnitudes ( $\Delta=$ final value - initial value) will also be calculated $[35,36]$ to verify the differences between the initial and final time points.

Table 1 Time points for outcomes

\begin{tabular}{|c|c|c|c|c|c|c|c|}
\hline \multirow[t]{3}{*}{ Outcomes } & & & \multicolumn{5}{|l|}{ Time point } \\
\hline & & & \multirow[t]{2}{*}{ Initial assessment } & \multirow[t]{2}{*}{ Final evaluation } & \multicolumn{3}{|l|}{ During the sessions } \\
\hline & & & & & Before and at the end & Every 5 min & 15 min after \\
\hline \multirow[t]{4}{*}{ Primary } & \multirow[t]{4}{*}{ Load monitoring } & Borg & & & $x$ & $x$ & $x$ \\
\hline & & $\mathrm{HR}$ & & & $x$ & $x$ & $x$ \\
\hline & & rMSSDmean & & & $x$ & $x$ & \\
\hline & & rMSSDcv & & & $x$ & $x$ & \\
\hline \multirow[t]{7}{*}{ Secondary } & ANS & HRV & $x$ & $x$ & $x$ & $x$ & \\
\hline & \multirow{4}{*}{$\begin{array}{l}\text { Cardiorespiratory } \\
\text { metrics }\end{array}$} & $\mathrm{BP}$ & $x$ & $x$ & $x$ & & \\
\hline & & $H R$ & $x$ & $x$ & $x$ & & \\
\hline & & $f$ & $x$ & $x$ & $x$ & & \\
\hline & & $\mathrm{SatO}_{2}$ & $x$ & $x$ & $x$ & & \\
\hline & Pain & VAS & $x$ & $x$ & $x$ & & \\
\hline & SPE & Borg & $x$ & $x$ & & & \\
\hline
\end{tabular}

SPE subjective perception of effort, $H R$ heart rate, $A N S$ autonomic nervous system, $H R V$ heart rate variability, $B P$ blood pressure, $V A S$ visual analog scale, $f$ respiratory rate, $\mathrm{SatO}_{2}$ oxygen saturation, $r M S S D$ parasympathetic modulation index, $r M S S D$ mean weekly mean of rMSSD expressed in milliseconds, $r M S S D c v$ weekly intra-individual coefficient of variation of rMSSD 
To analyze weekly differences in training loads using the SPE, HR, and HRV indices (ln rMSSDweekly and ln rMSSDcv) over the 12-week study duration, magnitudebased inferences will be calculated [36].

For ln rMSSDweekly, the smallest worthwhile change was set at 3\% [37]. For SPE, HR, and ln rMSSDcv, the smallest worthwhile change is defined as $0.2 \times$ betweensubject standard deviation. The quantitative chances to find differences in the variables to be tested will be assessed qualitatively as follows: $<1 \%$ almost certainly not, $1-5 \%$ very unlikely, $5-25 \%$ unlikely, $25-75 \%$ possible, $75-95 \%$ likely, $95-99 \%$ very likely, and $>99 \%$ certain. If the chances of having higher and lower results are both $>5 \%$, the true difference was assessed as nuclear [35]. The magnitude of the effect size will be qualitatively interpreted using the following thresholds: $<0.2$ trivial, 0.2-0.6 small, 0.6-1.2 moderate, 1.2-2.0 large, $2.0-4.0$ very large, and $>4.0$ nearly perfect [35].

To determine the correlations (dose-response) between ln rMSSD, HR, and SPE, Pearson's or Spearman's correlation will be used according to the normality of the data. The threshold used to evaluate the correlations quantitatively will be based on Hopkins [35] using the following criteria: <0.1 trivial, $0.1-0.3$ small, $0.3-0.5$ moderate, $0.5-0.7$ large, $0.7-0.9$ very large, and $>0.9$ almost perfect.

The cutoff points for load increments with the HRV indices and SPE were obtained from the receiver operating characteristic curve. The sensitivity, specificity, positive predictive value, and negative predictive value for each level of the Pilates method will also be calculated. The area under the curve will be considered significant when values $\geq 0.65$ are obtained [38]. In addition, a comparison will be made between the receiver operating characteristic curves of each HRV index to detect the most representative for determining the internal training load of the Pilates method.

The statistical analysis will be carried out with the Statistical Package for the Social Sciences, version 15.0 (SPSS Inc., Chicago, IL) and MedCalc Software bvba, version 14.10.2 (Oostende, Belgium). All data will be entered into the software twice. All participants included in the initial assessment will be considered under the intention-to-treat approach in which the worst outcome obtained by the study group will be selected to ensure the power of the data analysis.

\section{Discussion}

Previous systematic reviews have found a beneficial effect of Pilates on risk factors for various diseases, as well as positive responses in outcomes such as strength, flexibility, low back pain, and quality of life, among others $[2,39,40]$. However, the low methodological quality of previous studies and the lack of load monitoring of the exercises do not allow definitive conclusions to be drawn about the effect of the Pilates method. Moreover, there have been few studies investigating the effects of Pilates on the cardiac autonomic nervous system.

Methodological characteristics commonly found in studies published in the literature include lack of concealed allocation, no intention-to-treat analysis, unblinded assessors, lack of standardization of training on biological principles, and low familiarization of participants with the method. This study is a parallel randomized clinical trial to measure the effectiveness of the Pilates method on the cardiac autonomic nervous system and, mainly, to analyze methods of monitoring training loads.

The best measures for quantifying the internal load are SPE and HR, because they are cheap and simple to use. They can be measured by any healthcare professional. HRV is also important as an early and sensitive indicator of health impairment; however, correct analytical use of this tool requires detailed knowledge.

The training protocol described in this study was based on the most recent literature. A strength of the study is the parallel randomized clinical trial design with an intention-to-treat analysis. Moreover, the protocol is easy to replicate. However, a limitation is the inability to blind participants to their allocation.

This study may contribute by providing further information on the real autonomic benefits provided by this type of physical exercise and can be used to guide practitioners in prescribing the method, as well as offering more support to practitioners.

Note that this study uses the checklist of items for protocol studies to minimize bias. It was prospectively registered. The outcomes will be disseminated through publications in scientific journals and presentations at area congresses.

\section{Trial status}

The registration number is NCT03232866. The study start date was 1 September 2018. The primary completion date was May 2019. Patient recruitment is completed. The data were collected in June 2019. Altogether, 58 volunteers were assessed for eligibility, of whom four were excluded, so that 54 participants were randomized. All participants are attending ongoing study groups. The study completion date is December 2019.

\section{Additional files}

Additional file 1: Pilates exercises. (TIF $5581 \mathrm{~kb}$ )

Additional file 2: Pilates exercise sequences. (DOCX $17 \mathrm{~kb}$ )

Additional file 3: SPIRIT 2013 Checklist: Recommended items to address in a clinical trial protocol and related documents. (DOC $125 \mathrm{~kb}$ )

\section{Abbreviations}

ANS: Autonomic nervous system; FCT/UNESP : Center for Studies and Assistance in Physiotherapy and Rehabilitation of the Universidade Estadual Paulista; HR: Heart rate; HRV: Heart hate variability; rMSSD: Root mean-square 
of successive differences of adjacent RR intervals; rMSSDcv: Weekly intraindividual coefficient of variation of rMSSD; rMSSDmean: Weekly mean of rMSSD expressed in milliseconds; $\mathrm{SatO}_{2}$ : Oxygen saturation; SMS: Short Message Service; SPE: Subjective perception of effort; VAS: Visual analog scale

\section{Authors' contributions}

APSC and FMV were responsible for the study design. APSC, EPJ, AFM, TMB, CMP, and FMV commented on various versions of this study protocol. APSC, $E P J, T M B, A F M$, and FMV will be involved in recruiting participants and collecting data. All authors approved the final manuscript.

\section{Funding}

APSC was funded by the Coordenação de Aperfeiçoamento de Pessoal de Níve Superior, Brazil (CAPES) under finance code 001 and FMV by the Fundação de Amparo à Pesquisa do Estado de São Paulo (FAPESP, protocol 2017/20193-9).

\section{Availability of data and materials}

Not applicable.

\section{Ethics approval and consent to participate}

Ethics approval was granted by the ethics committee of FCT/UNESP (061942/2017). Informed consent will be obtained from all study participants.

\section{Consent for publication}

The patient consented to the publication of his images in Additional file 1.

\section{Competing interests}

The authors declare that they have no competing interests.

\section{Author details}

${ }^{1}$ Postgraduate Program in Physiotherapy, Universidade Estadual Paulista (FCT/UNESP), Presidente Prudente, SP, Brazil. Faculdade de Ciências e Tecnologia - FCT/UNESP, Departamento de Fisioterapia, Rua Roberto Simonsen, 305 - Cidade Universitária, Presidente Prudente, SP 19060-900, Brazil. ${ }^{3}$ Graduate in Physiotherapy, Universidade Estadual Paulista (FCT/ UNESP), Presidente Prudente, SP, Brazil. ${ }^{4}$ Department of Physiotherapy, Universidade Estadual Paulista (FCT/UNESP), Presidente Prudente, SP, Brazil.

\section{Received: 20 February 2019 Accepted: 25 August 2019}

\section{Published online: 17 October 2019}

\section{References}

1. Owsley A. An introduction to clinical Pilates. Athl Ther Today. 2005:10(4):19-25.

2. Bernardo LM. The effectiveness of Pilates training in healthy adults: an appraisal of the research literature. J Bodyw Mov Ther. 2007;11(2):106-10.

3. Rodrigues LB, Forjaz CL, Lima AH, Miranda AS, Rodrigues SL, Cardoso CG Jr, et al. A single bout of resistance exercise does not modify cardiovascular responses during daily activities in patients with peripheral artery disease. Blood Press Monit. 2014;19(2):64-71.

4. Prinsloo GE, Rauch HG, Derman WE. A brief review and clinical application of heart rate variability biofeedback in sports, exercise, and rehabilitation medicine. Phys Sportsmed. 2014;42(2):88-99.

5. Task Force of the European Society of Cardiology of the North American Society of Pacing Electrophysiology. Heart rate variability: standards of measurement, physiological interpretation and clinical use. Circulation. 1996; 93(5):1043-65.

6. Foster C, Florhaug JA, Franklin J, Gottaschall L, Hrovatin LA, Parker S, et al. A new approach to monitoring exercise training. J Strength Cond Res. 2001; 15(1):109-15.

7. Aubert AE, Seps B, Beackers F. Heart rate variability in athletes. Sports Med. 2003;33(12):889-919.

8. Ciolac EG, Mantuani SS, Neiva CM, Verardi CEL, Pessôa-Filho DM, Pimenta L. Rating of perceived exertion as a tool for prescribing and self regulating interval training: a pilot study. Biol Sport. 2015;32(2):103-8.

9. Azevedo A, Matos LF, Nakamura FY, Pereira G. Perception of effort monitors internal load during compounded circuit training. Motriz: Rev Educ Fís. 2016;22(1):90-3.

10. Woodcock J, Franco OH, Orsini N, Roberts I. Non-vigorous physical activity and all-cause mortality: systematic review and meta-analysis of cohort studies. Int J Epidemiol. 2011;40(1):121-38.
11. Orrow G, Kinmonth AL, Sanderson S, Sutton S. Effectiveness of physical activity promotion based in primary care: systematic review and metaanalysis of randomised controlled trials. BMJ. 2012;344:e1389.

12. Chan AW, Tetzlaff JM, Altman DG, Laupacis A, Gotzsche PC, Krle A-Jeric K, et al. SPIRIT 2013 Statement: defining standard protocol items for clinical trials. Rev Panam Salud Publica 2015:38(6):506-514.

13. Yamato T, Maher C, Saragiotto B, Moseley A, Hoffmann T, Elkins M, et al. The TIDieR checklist will benefit the physical therapy profession. Braz J Phys Ther. 2016;20(3):191-3.

14. Yamato TP, Maher CG, Saragiotto BT, Moseley AM, Hoffmann TC, Elkins MR, et al. The TIDieR checklist will benefit the physiotherapy profession. Physiother Can. 2016;68(4):311-2.

15. Treweek S, Lockhart P, Pitkethly M, Cook JA, Kjeldstrom M, Johansen M, et al. Methods to improve recruitment to randomised controlled trials: Cochrane systematic review and meta-analysis. BMJ Open. 2013:3:e002360.

16. Machado AF, Almeida AC, Micheletti JK, Vanderlei FM, Tribst MF, Netto Junior J, et al. Dosages of cold-water immersion post exercise on functional and clinical responses: a randomized controlled trial. Scand J Med Sci Sports. 2017:27(11):1356-63.

17. Broatch JR, Petersen A, Bishop DJ. Post-exercise cold-water immersion benefits are not greater than the placebo effect. Med Sci Sports Exerc. 2014 46(11):2139-47.

18. Tanaka H, Monaha KD, Selas DR. Age-predicted maximal heart rate revisited. J Am Coll Cardiol. 2001;37(1):153-6.

19. Moreira A, Bilsborough JC, Sullivan CJ, Ciancosi M, Aoki MS, Coutts AJ. The training periodization of professional Australian football players during an entire AFL season. Int J Sports Physiol Perform. 2015;10(5):566-71.

20. Edwards S. High performance training and racing. In: The Heart Rate Monitor Book. 8th ed. Sacramento: Feet Fleet Press; 1993.

21. Flatt AA, Esco MR, Nakamura FY. Individual heart rate variability responses to preseason training in high level female soccer players. J Strenght Cond Res. 2017:31(2):531-8.

22. Nakamura FY, Pereira LA, Rabelo FN, Flatt AA, Esco MR, Bertollo M, et al. Monitoring weekly heart rate variability in futsal players during the preseason: the importance of maintaining high vagal activity. J Sports Sci. 2016:34(24):2262-8.

23. Vanderlei LCM, Silva RA, Pastre CM, Azevedo FM, Godoy MF. Comparison of the Polar S810i monitor and the ECG for the analysis of heart rate variability in the time and frequency domains. Braz J Med Biol Res. 2008;41(10):854-9.

24. Marães VFSR, Teixeira LCA, Catai AM, Milan LA, Rojas FAR, Oliveira L, et al. Determinação e avaliação do limiar de anaerobiose a partir de métodos de análise da frequência cardíaca de sua variabilidade. Rev Soc Cardiol Estado de São Paulo. 2003:13(4):1-6.

25. Laborde S, Mosley E, Thayer JF. Heart Rate Variability and Cardiac Vagal Tone in Psychophysiological Research - Recommendations for Experiment Planning, Data Analysis, and Data Reporting. Front Psychol. 2017:8:213.

26. Shaffer F, Ginsberg JP. An overview of heart rate variability metrics and norms. Front Public Health. 2017;5:258.

27. Tulppo MP, Makikallio TH, Takala TES, Seppanen T, Huikuri HV. Quantitative beat-to-beat analysis of heart rate dynamics during exercise. Am J Phys. 1996:271(7):H244-52.

28. Smith AL, Reynolds $\mathrm{KJ}$, Owen $\mathrm{H}$. Correlated Poincaré indices for measuring heart rate variability. Australas Phys Eng Sci Med. 2007:30(4):336-41.

29. Irwin S, Tecklin JS. Fisioterapia cardiopulmonar. 3rd ed. São Paulo: Manole; 2003. p. 348-9.

30. Nunes WA, Terzi RGG. Pulse oximeters in the evaluation of oxygen transportation in critical patients. Rev Latino-Am Enfermagem. 1999; 7(2):79-85.

31. Malachias MVB, Souza WKSB, Plavnik FL, Rodrigues CIS, Brandão AA, Neves MFT, et al. 7a Diretriz brasileira de hipertensão arterial. Rev Soc Bras Cardiol. 2016;107(3):1-03.

32. Sellwood KL, Brukner P, Williams D, Nicol A, Hinman R. Ice-water immersion and delayed-onset muscle soreness: a randomised controlled trial. $\mathrm{Br}$ Sports Med. 2007:41(6):392-7.

33. Baroni BM, Leal Junior EC, De Marchi T, Lopes AL, Salvador M, Vaz MA. Low level laser therapy before eccentric exercise reduces muscle damage markers in humans. Eur J Appl Physiol. 2010;110(4):789-96.

34. Barbosa MPR, Netto Júnior J, Cassemiro BM, de Souza NM, Bernardo AF, da Silva AK, et al. Impact of functional training on cardiac autonomic modulation, cardiopulmonary parameters and quality of life in healthy women. Clin Physiol Funct Imaging. 2016;36(4):318-25. 
35. Hopkins WG. Measures of reliability in sports medicine and science. Sports Med. 2000;30(1):1-5

36. Batterham AM, Hopkins WG. Making meaningful inferences about magnitudes. Int J Sports Physiol Perform. 2006;1 (1):50-7.

37. Buchheit M. Monitoring training status with HR measures: Do all roads lead to Rome? Front Physiol. 2014;73(5):1-19.

38. Pivatelli FC, Santos MA, Fernandes GB, Gatti M, Abreu LC, Valenti VE, et al. Sensitivity, specificity and predictive values of linear and nonlinear indices of heart rate variability in stable angina patients. Int Arch Med. 2012;5(1):31.

39. Wells C, Kolt GS, Bialocerkowski A. Defining Pilates exercise: A systematic review. Complement Ther Med. 2012;20(4):253-62.

40. Yamato TP, Maher CG, Saragiotto BT, Hancock MJ, Ostelo RW, Cabral CM, et al. Pilates for low-back pain. Cochrane Database Syst Rev. 2015:7:CD010265.

\section{Publisher's Note}

Springer Nature remains neutral with regard to jurisdictional claims in published maps and institutional affiliations.

Ready to submit your research? Choose BMC and benefit from:

- fast, convenient online submission

- thorough peer review by experienced researchers in your field

- rapid publication on acceptance

- support for research data, including large and complex data types

- gold Open Access which fosters wider collaboration and increased citations

- maximum visibility for your research: over $100 \mathrm{M}$ website views per year

At BMC, research is always in progress.

Learn more biomedcentral.com/submissions 